\title{
Les Ordonnances sur la Médecine de 1569, première réglementation, à Genève, des rapports entre le médecin, la société et l'Etat
}

Par Roger Mayer

\section{Le médecin, la société et l'Etat}

Le problème des rapports entre le médecin, la société et l'Etat est devenu au cours du $20^{\mathrm{e}}$ siècle d'une acuité toute particulière et ceci en raison d'un ensemble de circonstances techniques, économiques, sociales et politiques. Ce problème a cependant existé de tout temps et nombreux sont les auteurs - de l'Antiquité jusqu'à nos jours - qui ont exprimé quelque opinion sur ce thème. Il serait intéressant d'en faire l'étude à travers les civilisations et les siècles et de suivre ainsi l'évolution qui s'est faite au cours de l'histoire, de la situation du médecin dans la société, en fonction de la structure même de cette société et face à l'Etat politique dont l'influence, grande ou petite, en conditionne les interactions.

Sur le simple plan des associations d'idées, certaines confrontations verbales permettent déjà de prendre conscience des influences réciproques qui existent entre ces trois termes, «médecin», «société», «Etat» : on parle en effet volontiers de «socialisation de la médecine» ou «d'étatisation de la médecine», mais on dira aussi «médicalisation de la société» et on parle également de «médecine sociale » et de l'«Etat social».

Une analyse exhaustive de cette relation triangulaire devrait comprendre une approche non seulement historique mais aussi économique et politique et devrait être complétée par une étude philosophique du problème. Notre propos est plus modestement centré sur un sujet qui restera historique et local : la genèse, au $16^{\mathrm{e}}$ siècle, à Genève, de la première réglementation entre le médecin, la société et l'Etat. Cette étude se terminera par quelques considérations sur ce qu'en fut l'évolution au cours des siècles suivants.

\section{Genève au $16^{e}$ siècle}

Pour situer cette création originale dans son juste contexte, il nous faut, très sommairement, évoquer ce qu'était Genève au milieu de ce $16^{\mathrm{e}}$ siècle. Nous en brosserons donc un bref tableau politique, économique et social. Ce tour d'horizon rapide et nécessairement simplifié est néanmoins utile pour situer dans quelle atmosphère y sont nées, en 1569, les «Ordonnances sur l'estat de la Médecine, Pharmacie et Chirurgie». 
Souvent, l'histoire se répète. Notre ville à cette époque, après une période de relative opulence au siècle précédent, subit une brusque crise économique, puis revient à nouveau, après la Réforme, à une certaine prospérité.

Au siècle précédent, au $15^{\mathrm{e}}$ siècle, Genève a donc vécu une période généralement favorable, malgré une situation de politique extérieure souvent tendue. La prospérité y est accentuée par l'importance croissante des célèbres foires, qui existent depuis le $13^{\mathrm{e}}$ siècle, mais atteignent alors leur apogée. Cette situation privilégiée change brusquement lorsqu'en octobre 1462, Louis XI (et son beau-père le Duc de Savoie) interdisent à leurs sujets de fréquenter les foires de Genève et que simultanément Louis XI donne un immense essor aux foires de Lyon, dont il fait de plus coïncider les dates avec celles de Genève. En quelques années, c'est la ruine pour Genève.

Le $16^{\mathrm{e}}$ siècle va être, lui, pour la cité, une époque riche en contrastes : il débute donc par une période économique très sombre qui va encore être aggravée - dans un premier temps - par l'adoption de la Réforme, isolant Genève plus fortement de ses voisins immédiats. Mais au milieu du siècle un revirement de la situation va se produire, paradoxalement causé lui aussi - et dans un deuxième temps - par la Réforme, le premier Refuge et leurs conséquences. En effet l'arrivée à Genève de nombreux réfugiés protestants, si elle y a momentanément augmenté la pauvreté, a ensuite été très favorable à l'économie. Les réfugiés qui se fixent à Genève doivent être logés et logés dans les murs de la ville, ce qui entraine une importante reprise d'activité dans la construction : on agrandit les maisons, on en construit dans les jardins et dans les cours et on rehausse les immeubles partout où cela est possible. D'autres secteurs économiques suivent. L'artisanat, enrichi de techniques nouvelles que les immigrés ont apportées avec eux devient de plus en plus prospère. Citons simplement comme exemples la confection du velours, la fabrication de rubans, l'industrie de la soie, puis de l'horlogerie, ainsi que les travaux des métaux précieux et l'orfèvrerie. L'imprimerie est déjà introduite à Genève au siècle précédent par les Allemands, mais l'arrivée d'imprimeurs français et surtout d'éditeurs va lui donner une importance de premier ordre dans ce domaine en raison des textes de doctrine réformée qui vont y être imprimés.

Après l'expansion de l'artisanat, c'est l'essor des professions libérales et c'est, dès le milieu du $16^{\mathrm{e}}$ siècle, la prospérité d'antan recouvrée. Pour être complet il nous faut aussi indiquer la contrepartie que cette croissance économique occasionne : parallèlement à l'expansion se développe une importante inflation qui devient même insupportable dans la deuxième partie du siècle et qui va entraîner une période de vie chère. C'est alors que le prêt à intérêt se répand, avec des taux qui vont s'élever à 8 , puis 10 , puis $12 \%$ et plus, ce qui est la raison pour laquelle 
la Compagnie des pasteurs est amenée à s'en préoccuper et va demander au Conseil de réglementer cette activité. Des troubles sociaux apparaissent, qui vont déclencher de la part de l'Etat la promulgation d'un certain nombre de réglementations professionnelles. Ce sont des conflits chez les potiers d'étains en 1537, puis chez les orfèvres en 1566. En 1559 de graves dissensions éclatent chez les imprimeurs, amenant l'intervention du Conseil. La profession va alors être très vite réglementée et surveillée par le Conseil et les ministres du culte, mais également très soigneusement protégée, «attendu que les impressions qui sortent de ceste cité ont beaucoup d'authorité partout où il y a des fidèles». Cela aura comme conséquence la rédaction d'un texte qui sera le premier réglement corporatif de Genève.

En ce milieu du $16^{\mathrm{e}}$ siècle, c'est aussi l'époque où sont mises en vigueur les diverses réglementations qui vont codifier dans les moindres détails la vie quotidienne de l'époque. Rappelons que les «Ordonnances ecclésiastiques» datent du retour de Calvin (1541), qu'elles sont renouvelées en 1561, avec l'adjonction de toute une législation familiale et matrimoniale et que les très fameuses «Ordonnances somptuaires» réglementant la façon de se vêtir datent de 1558 et qu'elles seront ultérieurement renouvelées et complétées à plusieurs reprises. Enfin, en 1577, des ordonnances encore plus précises et plus détaillées aggravent la sévérité des restrictions personnelles.

Nous comprenons alors - pour toutes ces raisons - combien il était surprenant, en ce milieu du $16^{\mathrm{e}}$ siècle, qu'une activité aussi importante pour la collectivité que l'art médical, ait encore échappé à toute réglementation.

\section{La médecine à Genève au $16^{e}$ siècle}

Avant la Réforme il n'y a guère de problèmes, car il n'y a que trois médecins à Genève et après 1542, date supposée de la mort de Patron ${ }^{1}$, ils ne seront plus que deux pendant quelques années! Mais parallèlement aux modifications démographiques et économiques que nous avons évoquées ci-dessus, on voit la situation évoluer dans la seconde partie du siècle. Après la mort de Calvin le nombre des médecins va beaucoup augmenter, puisqu'il s'élèvera à 15 à la fin du siècle. Notons - en regard de ces chiffres - que la population est estimée à environ 10000 âmes en 1537 (après la destruction des faubourgs qui avait déjà fait augmenter le nombre d'habitants dans la cité), alors qu'en 1589 on en compte à Genève 13 000. Les médecins nouveaux venus sont ou Français ou Italiens et leur situation dans la cité comme leur importance sociale ne sont pas très faciles à évaluer. Ils semblent avoir eu la parfaite confiance et l'estime de toute la population. Cepen- 
dant ils ne jouent encore aucun rôle politique important, bien que quelques-uns aient pu être élus au Conseil des Deux Cents.

On remarque toutefois que leur avis est souvent sollicité par le Conseil pour différents problèmes relatifs à la santé publique. Rappelons ici un petit fait qui concerne la construction du nouveau Collège. «Il fauldra dresser collège » avait écrit Calvin dans les «Ordonnances ecclésiastiques». ${ }^{2}$ La décision prise, une question primordiale se pose, celle de l'emplacement adéquat et de la conception générale de l'édifice. Une commission officielle est nommée pour cette tâche, composée de syndics et de quelques autres personnalités compétentes, au nombre desquelles figure un médecin, Philibert Sarasin ${ }^{3}$. Il semble que cette commission ait bien rempli son mandat: l'appréciation générale est favorable et l'endroit choisi - la colline de vignobles dite «des hutins Bolomier» - est approuvé, «d'autant que ce lieu est au beau regard et salubre aux estudiants », lit-on dans les registres du Conseil ${ }^{4}$.

Ils obtiennent aussi des faveurs particulières. En 1556, les médecins de Genève qui semblent bien n'avoir jamais beaucoup apprécié l'obligation du guet et de l'excharguet ${ }^{5}$ et qui avaient souvent demandé à en être libérés - obtiennent sur ce point un avantage partiel : il leur sera désormais possible de s'y faire remplacer, en payant un homme à leurs frais. Nous verrons comment, ultérieurement, ils obtiendront le privilège de s'en faire dispenser totalement.

Notons qu'en ce milieu du $16^{\mathrm{e}}$ siècle - avant même la promulgation des ordonnances sur la médecine - la pratique médicale n'était, en principe, pas totalement libre et qu'un médecin reçu habitant qui se proposait de s'installer à Genève devait, théoriquement, se soumettre à un examen. Cette formalité devait avoir lieu devant ses confrères et probablement aussi en présence de délégués du Conseil et de la Compagnie des pasteurs, mais ne semble pas avoir été exigée de façon régulière.

Par ailleurs, c'est à la même époque qu'eurent lieu les premières tentatives d'organiser un enseignement de la médecine, enseignement qui aurait ainsi pu être rattaché à l'Académie qui venait de naître. Ces tentatives furent toutes suivies d'échecs et ce n'est que trois cents ans plus tard que notre Faculté de médecine pourra officiellement voir le jour, non sans qu'il n'y ait encore eu quelques nouveaux essais, au $18^{\mathrm{e}}$ siècle, également sans suite et ceci pour diverses raisons où l'amour-propre de certains praticiens, la jalousie des autres, ne sont pas sans avoir joué un rôle certain.

\section{La genèse des Ordonnances sur la médecine}

Nous avons vu que vers 1540 , un médecin désireux de pratiquer à Genève était censé passer un examen, mais que ce dernier était loin d'être exigé de façon sys- 
tématique. Par ailleurs, pour les deux autres professions médicales (la pharmacie et la chirurgie), la pratique était restée totalement libre.

Cette situation très libérale constituait une anomalie surprenante dans cette République de Genève, où tout - ou presque - était déjà réglementé. L'absence de législation dans ce domaine était non seulement anormale, mais permettait aussi de nombreux abus et pouvait même être dangereuse pour la population. En effet, de très nombreux empiriques proliféraient à Genève et y pratiquaient librement sans contrôle aucun. Nous n'en citerons qu'un ici, le plus connu d'entreeux, un nommé Giordano Jarouba, dont le nom mérite de passer à la postérité en raison du fait que ses activités et son comportement ont fortement contribué à rendre nécessaire une réglementation de la pratique médicale à Genève. Ce Jarouba, originaire de Sicile, était un personnage très actif, passablement remuant et qui, pendant plus de dix ans, en fit voir de toutes les couleurs aux honorables praticiens genevois. Le Conseil, sans cesse importuné par son activité, dut prendre d'innombrables fois des mesures à son endroit. Il dépassa les bornes le jour où, apposant dans la ville des affiches où il se faisait une publicité éhontée, il déclancha un immense scandale. Son exemple fut contagieux, d'autres charlatans l'imitèrent. La mesure était comble et c'est alors que le Conseil décida sérieusement de prévoir une réglementation de la pratique de la médecine.

Cette réglementation passa par trois phases successives : proposition, rédaction et adoption des ordonnances.

Le 26 septembre 1560 , on peut lire dans les registres du Conseil ${ }^{6}$ la première mention d'une commission nommée pour mettre fin au désordre existant: elle se compose de médecins uniquement et aucun membre du Conseil n'en fait partie :

«Médecins, chirurgiens, apothicaires.»

«A esté parlé que plusieurs d'iceulx se meslent d'exercer et pratiquer combien qu'ils ne soient experts, qu'est cause que plusieurs en souffrent dans leurs maladies, et affin qu'il y aye ordre et que ceux qui sont capables puissent en liberté exercer et aux aultres soyent faites remontrances, a esté arresté qu'on commet les Spect. Lois Beljaquet, François Chapuis, St-Razin [sic!, pour Sarasin] et St-Ravi pour y adviser et y mettre ordre. ${ }^{7}$

Cette première tentative de légiférer n'eut aucune suite immédiate. Six ans plus tard (!) le Conseil, en date du 14 juin 1566, prend un nouvel arrêté, en tout point semblable, dans ses intentions, au premier :

«Médecins, cirurgiens et apoticaires.»

«Pour ce qu'on entend que plusieurs se meslent de médicamenter et gastent beaucoup de gens par leur ignorance, Arresté qu'en la Chambre des comptes, on apèle ceux qui sont expers en tous lesdits estats pour y faire un bon réglement. ${ }^{8}$ 
C'est ainsi la formation d'une nouvelle commission, composée d'une façon plus complète que la première, puisqu'elle comprend cette fois-ci des membres de la Chambre des Comptes, des médecins, des pharmaciens et des chirurgiens. Ses travaux vont durer trois ans, mais sans vraiment aboutir, de nouveau, à quoi que ce soit de concret.

Le 22 avril 1569, un argument de plus se présente pour démontrer la nécessité et l'urgence de remettre en chantier la réglementation qui se fait attendre depuis bientôt neuf ans: c'est la découverte dans certaines pharmacies, qui n'étaient plus régulièrement contrôlées, de préparations vieillies et devenues toxiques :

«Pour ce qu'à faulte de revoir les boutiques des apoticaires, plusieurs peuvent avoir de vieles drogues et aultres falsifiées domageables aux corps humains, Arresté que les Srs. Aubert et Chasteauneuf assemblent les médecins, chirurgiens et apothicaires, pour adviser d'une bonne réformation. ${ }^{9}$

Dès lors les ordonnances vont être rapidement mises sur pied. En effet, une semaine après seulement, sur rapport des deux commissaires désignés, le gouvernement décide de réunir les trois corps concernés, qui sont convoqués pour faire valoir leurs propositions :

«29 avril 1569. Médecine.»

«Les Srs. commis ayant rapporté l'advis des médecins, cirurgiens et apoticaires sur la réformation de la médecine, d'autant que c'est chose importante et concernant la santé des corps, Aresté de les apeler tous à Lundy matin à 5 heures, pour les ouyr tous sur les difficultés qu'ils voudront amener. ${ }^{10}$

Personne ne fit défaut à ce rendez-vous matinal, où lecture fut faite du projet de loi rédigé par les membres des trois corporations intéressées :

«Lundy 2 de May. Le Conseil estant expressément assemblé et tous les médecins, cirugiens et apoticayres de la ville présentz, A esté faite lecture des articles par eux dressés pour la réforme de ?a médecine, sur chacun desquelz ont esté ouys ceux qui ont voulu remonstrer quelque chose. Arresté qu'en la Chambre des Comptes, on appelle les vieux médecins et chirurgiens, pour dresser le tout et soldre les difficultez affin d'en conclure puys après en Conseil. »11

Il faut souligner ici un aspect très remarquable de cette procédure : la rédaction de ce texte fondamental des "Ordonnances sur la Médecine», qui va codifier de façon précise tout ce qui importe à l'époque pour la pratique de l'art de guérir, si elle a bel et bien été ordonnée par le Conseil - donc par l'Etat - a été en revanche totalement effectuée, et en toute liberté, par les membres des trois corporations concernées. C'est un détail important qu'il est essentiel de relever.

Nous donnerons ici le texte complet des articles 1 à 10 des Ordonnances, articles qui concernent les médecins. Viennent ensuite douze articles qui réglementent la 
pratique des apothicaires et onze articles concernant les chirurgiens, que nous ne transcrirons pas car leur étude sortirait du cadre de notre sujet. Le texte datant de 1569 n'existant plus à l'état d'original, celui que nous donnons ci-dessous est celui du $17^{\mathrm{e}}$ siècle, extrait du Livre des Ordonnances de la Faculté de médecine ${ }^{12}$. La fig. 1 en présente la reproduction photographique de la première page. Il faut noter que le copiste de 1697 a modernisé l'orthographe du texte primitif du $16^{\mathbf{e}}$ et qu'à l'article deux, il a manifestement sauté quelques mots (voir la note No 13).

\title{
Ordonnances sur l'estat de la Médecine, Pharmacie et Chirurgie Passées en Conseil le XI $I^{e}$ May 1569
}

\begin{abstract}
Article $1^{\text {er }}$
Premièrement que désormais Nuls Médecins, Pharmaciens, ni Chirurgiens nouveaux venus n'entreprendront exercer l'art sans avoir donné preuve suffisante de leur savoir, soit pour étre gradués en université fameuse, ou avoir bon témoignage des lieux où ils auront exercé leur art, ou qu'ils soient auparavant que pratiquer examinés, les Médecins, par les Médecins, et les Apothicaires et Chirurgiens, par les trois Estats de Médecine en présence des Députés de la Seigneurie, étans trouvés capables leur seront données Lettres patentes, afin qu'ils soient tenus avec honneur au rang des autres.
\end{abstract}

2.

Que tous les Médecins, Apothicaires et Chirurgiens s'assembleront deux fois l'année, assavoir le premier $\mathrm{Me}[\mathrm{r}]$ credi de Septembre ${ }^{13}$ pour enquérir s'il y auroit quelque désordre en la Ville touchant leur art, ou ceux qui l'exercent pour remontrer et corriger le tout amiablement, présens lesdits Seigneurs Députés.

3.

Que les Médecins, Apothicaires et Chirurgiens dressent un dispensaire pour ceste ville et que ceux qui le dresseront se trouvent toutes les semaines une fois, en certain lieu et heure, là où pourront venir les autres Médecins, Apothicaires et Chirurgiens et dire leur advis sur ce qu'on aura travaillé toute la semaine, puis sera signé et approuvé par tous les Médecins, Apothicaires et Chirurgiens demeurans en ceste ville et mis en lumière.

4 .

Que tous les ans soit choisi un Médecin qui lise quelques mois de l'année aux Chirurgiens et qui fasse anatomies publiques avec un Chirurgien, et un autre qui 
lise l'hyver aux Apothicaires, et se fasse ladite élection par la plus grand'voix desdits trois Estats, présents lesdits Seigneurs Commis, sans prétendre aucun salaire de la Seigneurie.

5 .

Que les Médecins entreux tiennent l'ordre et le rang accoutumé afin d'éviter confusion et querelles aux consultations et assemblées: c'est que les derniers venus en la ville parlent les premiers en montrant, horsmis que celui qui aura pansé le malade proposera le premier la maladie. Le mesme ordre soit observé des Apothicaires et Chirurgiens, mais quand ils seront appellés par la Seigneurie, les premiers assis parleront devant.

6.

Que nul Médecin, encores qu'il entende la Pharmacie et Chirurgie, ne s'ingère d'appliquer cataplasmes, unguens, emplastres et autres remèdes requérans opération manuelle, ne faire unguent de verolle, ne faire d'icelle suer, ni aussi exécuter les opérations manuelles sans l'assistance d'un Chirurgien, si ce n'estoit pour son mesnage particulier.

7.

Que nul Médecin ne s'ingère de faire ou exhiber aucun médicament sans Apothicaire, seulement se contenteront de prescrire chez le malade la raison de vivre.

8.

Que outre les Médecins députés pour le grand hospital et pour les povres estrangers, chacun Médecin soit tenu visiter les povres malades du quartier auquel il habitera, estant requis par aumosne.

9.

Que les médecins et Chirurgiens en leurs receptes soient tenus écrire le nom pour qui, la datte, et signer les susdites receptes.

10.

Que tous médecins advertissent des maladies suspectes.

L'ultime révision prévue dans le rapport du Conseil du 2 mai est alors rapidement menée et neuf jours après, les ordonnances sont définitivement adoptées par le Conseil. On lit en date du 11 mai 1569 : 
(1)

-ncienules Ordonnances - Sur lesstar de la Medecine. Tharmacic at Chirurgie, Passécs en Conseil le xi Mayis69. Article i: $(5 r$ Pharmaciens, ni Chinurgiens nouseaux venus néntreprendront exercer lárt sans auoir donne preuse ruffisante de leur savoir, soit pour étre qradues. en uniuersite famcuse), ou auoir bon temoignage des lieux ou its auront cocerce leur art, ou quits soient auparauant que pratiquer examines, les Medecins, par los Medecins, et las Apothicaires et Chirurgiens, par les troij Estats de thedecine en presence Des Deputes. de la. Signcuric, étans tromes capabtes leur. serone Donnecs Lettres patentes, afin quils soient tenus auec honneur au rang des autres.

\section{2:}

()

ULC tous les Mndecins, Apothicaires et Chiruryiens - ássembleront deux fois lánne, assauoir le premier. Mecredi de. Septembre pour enquerir. Sil y aurdit quelque desordre en la ville touchant leur art, ou caux qui Lixercent pour remontrer et corriger le tout amiablement prescns Lesdits. Secgneur. De Cutes.

Fig. 1. Ordonnances sur la Médecine. Première page de la copie du $17^{\mathrm{e}}$ siècle 
«Médecine. Les ordonnances sur la Médecine, Pharmacie et Chirurgie ayant esté reveues à la Chambre des Comptes et derechefs icy prises en main, article par article, ont esté passées. Et ont esté les Srs. de la Maisonneuve et Aubert commis pour tenir main à l'observation desdites ordonnances. $\rangle^{14}$

\section{Commentaire des Ordonnances}

En reprenant successivement ces dix articles nous pouvons en résumer l'essentiel de la façon suivante :

L'art. 1. Etablit les conditions d'admission à l'agrégation.

L'art. 2. Crée une assemblée habilitée à connaître et à résoudre les problèmes d'éthique ou de déontologie touchant à la pratique médicale qui pourraient se présenter dans la ville.

L'art. 3. Prévoit la rédaction d'un «dispensaire», c'est à dire d'une pharmacopée. Une commission était prévue pour ce travail qui n'a finalement pas été exécuté. La Pharmacopoea Genevensis fut réalisée en fait deux siècles plus tard (en 1780) par trois médecins, les docteurs Daniel De la Roche, Charles-Guillaume Dunant et Louis Odier.

L'art. 4. Prévoit des cours que l'on pourrait appeler «d'enseignement continu» à l'usage des chirurgiens et des apothicaires.

L'art. 5. Enumère quelques règles de préséance. Elles sont actuellement bien désuètes, mais il faut se rappeler le rôle que jouait alors l'étiquette dans toutes les circonstances de la vie sociale de l'époque.

L’art. 6. Précise les compétences et délimite les prérogatives du médecin, quelle que soit l'étendue de sa formation, en ne lui permettant pas de pratiquer des opérations manuelles sans l'assistance d'un chirurgien.

L'art. 7. Interdit formellement au médecin de confectionner ou de vendre des médicaments.

L'art. 8. Prévoit l'assistance aux malades indigents, qui sera assurée - en plus des médecins spécialement désignés pour assurer les soins à l'hôpital ou auprès des "povres estrangers» - par chaque médecin dans son quartier, gratuitement.

L'art. 9. Donne des directives précises pour la rédaction par les médecins et les chirurgiens des prescriptions médicales, sur lesquelles doivent obligatoirement figurer le nom du malade, la date et la signature. L'obligation d'indiquer le nom pouvait poser en pratique de délicats problèmes en 
regard du secret professionnel. Elle donna lieu à de nombreuses discussions. Lors de la révision des ordonnances qui aura lieu en 1658, une exception sera prévue pour les cas de maladies vénériennes: «Les Docteurs-médecins composeront leurs ordonnances ou prescriptions [...] y ajoutant le nom de la personne pour laquelle chaque ordonnance est faite, sauf aux maladies secrètes.»

L'art. 10. Prévoit l'obligation pour le médecin d'informer l'Etat des «maladies suspectes», soit une obligation similaire à notre actuelle déclaration des maladies contagieuses. Cette obligation était importante à l'époque. Le gouvernement devait pouvoir être tenu au courant de la situation sanitaire de la cité, car en cas d'épidémie (de peste en particulier) des mesures préventives très rigoureuses étaient prévues. Outre les mesures de propreté, l'interdiction absolue de l'entrée de la ville aux personnes et aux marchandises était ordonnée et leur application était lourde de conséquences pour le commerce et pour l'économie locale. Il s'agissait donc de prendre ces mesures à bon escient et au bon moment et la responsabilité des médecins était grande en cette matière.

Nous n'avons pas transcrit et nous ne pourrons pas commenter ici les articles qui concernent les pharmaciens et les chirurgiens, malgré leur grand intérêt. Faisons toutefois une exception pour les deux premiers articles concernant les pharmaciens :

L'art. $1^{\text {er }}$ du réglement des apothicaires précise : «Que nul ne soit admis à la Maistrise s'il n'est Citoyen ou Bourgeois.» La concurrence était déjà vive chez les pharmaciens à cette époque et elle explique cet article restrictif. Une telle clause aurait été inapplicable aux médecins en 1569 (il y avait encore pénurie) et leur recrutement aurait été beaucoup trop restreint si on n'en avait pas ouvert l'accès aux étrangers. Nous verrons que lors de la révision de ces ordonnances, un siècle plus tard, en 1658, cette limitation de la pratique aux seuls citoyens ou bourgeois va être également prévue pour les médecins.

L'art. 2 ordonne: «Que, deux ou trois fois l'an, les boutiques des Apothicaires soyent visitées à l'impourveu par deux Médecins, deux Apothicaires et les Commis de la Seigneurie, lesquels promettront par serment visiter les drogues et compositions en bonne conscience, sans haine ou faveur des uns ou des autres, et jetter là celles qui seront corrompues ou gastées afin qu'elles ne soient mises en usage $[\ldots] . »$

L'ensemble des articles des «Ordonnances sur la Médecine» constitue pour l'époque une législation remarquablement élaborée. La pratique ne suivant 
cependant pas toujours la théorie, on doit à la vérité de constater que cette réglementation ne fut pas appliquée avec un zèle extraordinaire: les membres des trois professions continuèrent à empiéter allègrement les uns sur les autres, la visite des officines des pharmaciens ne fut plus pratiquée régulièrement après quelques années et, à nouveau, des charlatans, empiriques ou autres illégaux de toute sorte se mirent à proliférer.

Notons encore que peu après l'adoption de ces ordonnances, il y fut apporté deux adjonctions qui méritent d'être signalées :

Le 24 mai 1569, les médecins obtiennent d'être déchargés - cette fois-ci totalement - de l'obligation du service du guet. La décision est motivée en soulignant le fait qu'ils «sont tenus de servir les paouvres en leur quartier gratuitement ». ${ }^{15}$ Cette faveur exceptionnelle est cependant partagée avec la corporation des imprimeurs, qui eux l'ont obtenue du fait qu'ils travaillent pour «la gloire et l'honneur de Dieu». Ce privilège fut d'ailleurs retiré aux médecins quelques années plus tard.

La seconde adjonction faite aux ordonnances concerne les apprentis pharmaciens pour lesquels il est précisé : «Que nul ne fust admis apprenti d'apoticaire qu'il ne sceust latin congru. ${ }^{16}$ Les prescriptions des médecins étant toujours rédigées en latin, la connaissance de cette langue était évidemment indispensable à la pratique de la pharmacie.

Mis à part les quelques remarques que nous avons faites plus haut concernant le sérieux avec lequel les ordonnances furent appliquées en pratique, il faut admettre que ces dernières n'en vont pas moins rester le texte fondamental qui va régir la médecine genevoise pendant presque un siècle.

\section{La révision des ordonnances de 1569}

Ce n'est qu'au milieu du $17^{\mathrm{e}}$ siècle - en 1658 - que va être adoptée une révision générale des ordonnances sur la médecine. Cette révision conservera l'esprit général de 1569 , en en rajeunissant la forme et en y ajoutant quelques dispositions nouvelles ${ }^{17}$. Nous ne citerons ici que les trois premiers articles de ces nouvelles ordonnances :

Art. $1^{\text {er }}$

Nul n'exercera aucune partie de la Médecine dans cette Cité s'il n'est agrégé au Corps de Médecine selon sa profession.

Art. 2.

Nul ne sera agrégé s'il n'est Citoyen ou Bourgeois. 
Art. 3.

Nul Docteur ou Maître n'exercera plus de l'une des trois professions en tout ou en partie, si ce n'est pour sa propre famille ou en cas de nécessité urgente.

Ce troisième article redit plus clairement encore qu'en 1569 la totale séparation des trois professions et l'incompatibilité de la pratique de l'une d'entre elles avec l'une quelconque des deux autres. Quant aux deux premiers articles, ils consacrent d'importantes modifications, car l'article premier fait dès lors du corps médical de Genève une corporation fermée, avec agrégation par cooptation et par ailleurs, l'article deuxième, ne permettant l'admission qu'aux Citoyens ou Bourgeois (nous avons vu qu'en 1569 seuls les pharmaciens subissaient cette limitation), démontre que la situation numérique des médecins avait certainement bien changé. Ce nouvel article avait un double avantage : pour les médecins, il limitait le nombre des candidats admissibles et pour l'Etat, il pouvait être une source de revenus supplémentaires en incitant les médecins désireux de pratiquer à Genève à se faire recevoir Bourgeois, ce qui revenait à payer à l'Etat une somme qui allait devenir de plus en plus importante au cours des siècles.

C'est ici l'occasion de relever un phénomène régulier que l'on retrouve au cours des siècles dans les relations entre le médecin, la société et l'Etat, à Genève et ailleurs et qui fait qu'en période de pénurie de médecin, l'Etat - qui se veut le garant de l'assistance médicale de la population - s'efforce d'attirer les praticiens en leur octroyant avantages et facilités diverses, alors que lorsque la pléthore médicale s'annonce, ce sont les médecins qui demandent à l'Etat le renforcement de dispositions légales restrictives, au niveau des études, des diplômes ou des conditions d'admission à la pratique.

Toutes ces situations peuvent devenir source de conflits. En voici un simple exemple, pris au $18^{\mathrm{e}}$ siècle : lorsque en 1766 , Genève dut subir - en raison des désordres de sa politique intérieure - la médiation de Berne, de Zurich et de la France, il fut précisé dans l'Edit de Médiation, plus exactement nommé le «Réglement de l'illustre Médiation pour la pacification de la République de Genève», ${ }^{18}$ que les natifs seraient désormais admis à exercer tous les métiers et qu'ils pourraient même accéder à la maîtrise. Le Conseil s'apprête donc à permettre la maîtrise de la pharmacie et de la chirurgie à des natifs! Beau tollé chez les membres des trois corps qui exposent avec véhémence au Conseil la différence qui existe entre les métiers, ou arts "mécaniques», ouverts aux natifs par l'Edit de Médiation et les arts «libéraux», dont il n'est pas question qu'ils le soient. Il en résulte une épreuve de force où le Conseil l'emportera. Sa décision ira même au delà de l'esprit de l'Edit de Médiation, puisque il va être alors clairement stipulé, 
le $1^{\text {er }}$ septembre 1766 , qu'une exception sera ajoutée à l'article deuxième des Ordonnances de 1658, qui précisera dès lors que «toutes personnes pourront être aggrégées à ce corps, quand même elles ne seroient ni citoyens ni bourgeois, lorsqu'elles en auroient obtenu l'agrément du Conseil, tant pour la médecine que pour la pharmacie et la chirurgie ${ }^{19}$

Sorti victorieux, le Conseil n'abusera pas de cette possibilité qui lui était ainsi donnée d'agréer même des étrangers et il se contentera d'en octroyer la faveur aux natifs et d'ailleurs de n'en faire usage qu'avec beaucoup de discrétion.

\section{L'évolution ultérieure des rapports entre le médecin, la société et l'Etat}

Nous avons exposé en détail ce que furent, à Genève, les «Ordonnances sur la Médecine» de 1569.

Nous avons préalablement vu dans quel contexte politique, économique et social elles ont vu le jour.

Nous en avons ensuite étudié successivement les différents articles réglant l'activité du médecin, laissant de côté ceux concernant les professions d'apothicaire et de chirurgien.

Nous avons vu comment elles ont été alors appliquées durant un siècle, puis comment elles furent modifiées en 1658 pour rester dès lors, pendant un nouveau siècle, la réglementation essentielle codifiant la pratique médicale à Genève.

Nous aimerions avant de conclure, évoquer encore brièvement comment ces rapports entre le médecin, la société et l'Etat se sont ensuite amplifiés et intriqués, pour parvenir à la complexité que nous leur connaissons aujourd'hui.

Très schématiquement, on pourrait dresser ainsi la liste des principales modifications qui se sont produites au cours des trois derniers siècles :

- C'est d'abord une réglementation toujours plus complète de la pratique médicale, créant un véritable droit à la pratique légale par opposition à la pratique illégale, ce qui, par voie de conséquence, va amener l'Etat à étendre ses prérogatives dans le domaine de la formation médicale, de la délivrance du diplôme (qui deviendra un diplôme d'Etat) et à réglementer les conditions de l'établissement du médecin.

- C'est aussi l'emprise de plus en plus forte de l'Etat dans l'important domaine de l'hygiène publique, où, après les soucis des grandes épidémies de lèpre, de peste ou de variole, les progrès de la prévention vont entraîner d'autres préoccupations, qui vont de l'extension des vaccinations, à la lutte contre les maladies vénériennes, la tuberculose ou l'alcoolisme. 
Dès le $17^{\mathrm{e}}$ siècle, on verra à Genève l'institution d'une «Chambre de Santé» dont la tâche sera l'étude de problèmes spécifiquement médicaux, puis d'une «Chambre de Netteté» pour ce qui concerne les problèmes que nous appellerions actuellement de la pollution et de l'environnement. Le nettoyage des rues de Genève n'était pas le plus petit d'entre-eux et il a fait couler beaucoup d'encre à chaque génération ${ }^{20}$.

En matière d'hygiène et de santé publique les situations les plus diverses se posent au médecin et à l'Etat, qui vont de la découverte - en 1620 - d'une source d'eau minérale à Cologny ${ }^{21}$, jusqu'à la réglementation de la vente des champignons au $20^{\mathrm{e}}$ siècle $^{22}$ !

- C'est également l'importance croissante des prérogatives de l'Etat dans le domaine hospitalier. L'histoire des hôpitaux de Genève est passionnante à étudier. Ce n'est pas notre propos de parler ici en détail du problème hospitalier, si ce n'est pour l'évoquer comme étant une situation typique où les intérêts médicaux de la société et de l'Etat sont nécessairement intimement liés. Le prodigieux développement technique de la médecine depuis le $19^{\mathrm{e}}$ siècle rend d'ailleurs ce sujet particulièrement important de nos jours.

- C'est encore l'apparition et le développement progressif, dès le $19^{\mathrm{e}}$ siècle surtout, d'une nouvelle discipline médicale - la médecine légale - où les rapports entre le médecin, la société et l'Etat vont trouver leur expression la plus étroite. Rappelons en passant que jusqu'à une époque relativement récente ces tâches faisaient partie des devoirs usuels du praticien. Au $19^{\mathrm{e}}$ siècle encore, dans une ville comme Genève, tout médecin pouvait être commis «médecin-légiste» et pouvait être appelé par l'autorité judiciaire à pratiquer pour l'Etat, constat de blessure, expertise ou autopsie médico-légale. La réquisition du médecin à ces fins était faite dans les formes en usage, ou l'élégance du style ne parvient cependant pas à masquer ce que la mission a d'impératif et d'obligatoire ! On en jugera par les quelques lignes suivantes, qu'un médecin genevois célèbre - Jean-Pierre Maunoir - reçoit le 22 juin 1827, sous pli urgent, porté par un commissionnaire et portant la simple suscription «Monsieur le Docteur Maunoir - En Ville» :

«Le Juge d'Instruction de la République et Canton de Genève, invite et en tant que besoin requiert Monsieur le Docteur Maunoir, conformément à la loi, de constater par procès-verbal les voies de fait et blessures exercées le 18 courant sur la personne du Sieur Jean Mallet [...] (signé) Fréd. A. Cramer. „23

- On constate également que les relations entre le médecin et l'Etat, au service de la société, sont aussi très étroites quand le médecin est l'employé de l'Etat ou qu'il est commis par lui pour une tâche spécifique. Il existe alors un lien de subordination qui est plus ou moins contraignant suivant les époques. Alors qu'actuel- 
lement on souligne volontiers la mainmise croissante de l'Etat sur l'activité des médecins («l'étatisation de la médecine»), remarquons simplement que cette notion devrait être nuancée et toujours replacée dans son contexte historique et politique pour être bien définie. A titre anecdotique, rapFelons que dans la Genève de la fin $d u 18^{\mathrm{e}}$ siècle, après l'annexion par la France ${ }^{24}$ et sous le Directoire, le médecin nommé «officier de santé Chirurgien des Pauvres» du Bureau municipal de Bienfaisance de la Commune de Genève devait prêter à l'Etat - et par devant le Commissaire du Directoire Exécutif ! - le serment suivant, "prescrit par les lois»: «Je jure haine à la Royauté et à l'anarchie, attachement et fidélité à la République française et à la Constitution de l'an trois; je promets de remplir avec exactitude et fidélité les fonctions de mon emploi [...]. ${ }^{25} \mathrm{Il}$ n'en est pas demandé autant au médecin du $20^{\mathrm{e}}$ siècle titulaire d'un poste officiel!

- On assiste enfin, dans la dernière partie du $19^{\mathrm{e}}$ siècle, à la création et au développement de caisses de secours mutuels en cas de maladies. Les associations médicales se structurent, au niveau cantonal d'abord, puis au niveau fédéral. L'Etat n'y demeure pas indifférent et va s'immiscer de plus en plus dans les rapports entre le médecin et le malade. Plus récemment, des assurances sociales centralisées au niveau fédéral sont créées et simultanément s'élabore une législation de médecine sociale extrêmement complexe. Parallèlement au progrès technique, le financement de la médecine, surtout hospitalière, devient - et ceci dans tous les pays - de plus en plus difficile.

Actuellement ces problèmes explosent. L'activité du médecin dans la société, sous toutes ses formes, est discutée publiquement. Les journaux quotidiens sont saturés de nouvelles médicales ou médico-sociales et plus que jamais les relations entre le médecin, la société et l'Etat sont à la première page de l'actualité. Mais ceci nous entraîne hors de notre sujet et devient une autre histoire...

\section{Conclusion}

Le texte des «Ordonnances sur la Médecine» de 1569, ordonnances qui sont à Genève la première réglementation officielle, ordonnée par l'Etat, de l'activité du médecin dans la société, méritait d'être rappelé ici. Dans leur sobre concision, ces ordonnances, rédigées alors librement par les membres de la corporation médicale, traitent l'essentiel des problèmes qui se posaient au $16^{\mathrm{e}}$ siècle et elles préfigurent toute la législation ultérieure, qui va progressivement se développer parallèlement à la complexité croissante des liens, qui au cours des siècles, se sont de plus en plus étroitement noués, entre le médecin, la société et l'Etat. 
Notes

${ }^{1}$ Patron, Pierre-Paul, de Milan. Reçu bourgeois gratis le 8 juillet 1505. Est un des médecins de Viret en 1535. Quitte ensuite Genève, puis est admis à y rentrer. Dernière mention le 29 août 1542 , alors qu'il était très malade.

${ }^{2}$ Nous transcrirons ici le passage intégral dans lequel se trouvent cette phrase de Calvin si souvent citée: "Mais pource qu'on ne peult proufiter de telles leçons que premièrement on ne soit instruict aux langues et sciences humaines, et aussi est besoing de susciter de la semence pour le temps advenir, affin de ne laisser l'église déserte à noz enfantz, il fauldra dresser collège pour les instruire, affin de les préparer tant au ministère que [au] gouvernement civil» (Ordonnances ecclésiastiques, 20 novembre 1541).

${ }^{3}$ Sarasin, Philibert, né à Charlieu en Charolais vers le commencement du XVI ${ }^{\mathrm{e}}$ siècle. Docteur en médecine à Perpignan. Reçu habitant le 24 août 1551. Bourgeois pour dix écus le 24 octobre 1555 . Succéda à Textor comme médecin de Calvin et le soigna jusque dans sa dernière maladie. Mort le 5 mai 1573.

${ }^{4}$ Donnons en encore deux extraits : "Icy est ordonné que les Seigneurs commis aux forteresses et aultres visitent le lieu qui leur paraistra le meilleur pour fere ung College [...]. Aussy qu'on face un pourtraict de ce qui sera advisé de fere, affin qu'on le voye bien avant que de conclure» (Reg. Conseil, 17 janvier 1558). «Icy est arresté que pour mettre main à l'instauration d'ung College, après disner, les sindiques aillent visiter le lieu vers Rive, avec les massons et chappuis [charpentiers], et qu'ils demandent à Mess. Calvin, Sarasin et aultres gens d'esprit, pour bien comprendre l'édifice, suyvant ce qu'il en a esté parlé cy-devant» (Reg. Conseil, vol. LIV, fol. 133, 25 mars 1558).

${ }^{5}$ Obligation de monter la garde et de faire des patrouilles de nuit.

${ }^{6}$ Les extraits des registres du Conseil qui suivent sont cités d'après Léon Gautier, La médecine à Genève jusqu'à la fin du dix-huitième siècle, Genève (Jullien et Georg) 1906.

${ }^{7}$ Reg. Conseil, vol. LVI, fol. 82, 26 septembre 1560.

${ }^{8}$ Reg. Conseil, vol. LXI, fol. 52, 14 juin 1566.

${ }^{9}$ Reg. Conseil, vol. LXIV, fol. 64, 22 avril 1569.

${ }^{10}$ Reg. Conseil, vol. LXIV, fol. 67, 29 avril 1569.

${ }^{11}$ Reg. Conseil, vol. LXIV, fol. 67, 2 mai 1569.

${ }^{12}$ Série des Manuscripts historiques No 28 bis (cité par Léon Gautier), actuellement Archives Etat de Genève, Santé F 1. p. 1-14.

${ }^{13} \mathrm{Il}$ y a manifestement ici une lacune. Une copie plus ancienne (Archives Etat de Genève, Industrie A 1), qui n'était pas connue de Léon Gautier, nous permet de reconstituer le texte primitif. Il faut lire: «le premier Mercredi de Mars et le premier Mercredi de Septembre».

${ }^{14}$ Reg. Conseil, vol. LXIV, fol. 71, 11 mai 1569.

${ }^{15}$ Reg. Conseil, vol. LXIV, fol. 77, 24 mai 1569.

${ }^{16}$ Reg. Conseil, vol. LXV, fol. 36, 2 mars 1570.

17 Reg. Conseil, vol. CLVIII, p. 56, 15 février 1658.

${ }^{18}$ Réglement de l'illustre Médiation pour la pacification de la République de Genève, article XXXVI.

${ }^{19}$ Reg. Conseil, vol. CCLXVII, p. 1016, $1^{\text {er }}$ septembre 1766.

${ }^{20}$ Quelques exemples seulement: le problème exista de tout temps. Deux articles de la Charte des Franchises de 1387 nous renseignent sur l'état des rues de Genève au $14^{\mathrm{e}}$ siècle : 
art. XLIV «Quiconque aura placé ou fait placer du fumier dans un chemin ou dans une rue publique, depuis la fête de Pâques jusqu'à la Toussaint, devra le faire ôter dans les trois jours [...]. Quiconque aura contrevenu, paiera trois sous de Genève si plainte a été déposée». Art. LXXI « Nul ne construira ou ne fera construire dans les rues des étables à porcs ». Mayor, J., L'ancienne Genève, Genève 1896. - Au $17^{\mathrm{e}}$ siècle, John Evelyn (1620 1706) le célèbre voyageur ne trouve que deux villes qui égalent Genève en saleté : Edimbourg et Lisbonne. - Enfin au début du $19^{\mathrm{e}}$ siècle, deux presque contemporains sont d'un avis quelque peu divergent dans leurs conclusions. C'est d'abord le Dr. J.-P.Maunoir (1768-1861) qui écrit au représentant du gouvernement la lettre suivante : «Monsieur le Syndic, Je viens soumettre à la sagesse de vos réflexions un sujet qui a un rapport immédiat avec la propreté de la ville \& qui regarde plus qu'on ne le pense la santé publique. Je veux parler du balayage des rues. L'abus qui règne dans cette partie bien secondaire de l'administration urbaine frappe moins nos concitoyens à cause de leur longue habitude de le voir, que les Etrangers, pour lesquels il est une vraie nouveauté. Voici le mal: on entasse au milieu des rues les immondice de toutes les maisons. La vue \& l'odeur de ces fumiers récents frappe aussi désagréablement les yeux que l'odorat; mais ce n'est rien auprès de l'espèce de supplice qu'éprouvent les gens à pied dans les moments où en été, $\&$ dans les temps de sécheresse, on balaye les rues. Il faut alors traverser des tourbillons de poussière infecte; c'est insupportable pour toute personne bien-portante, c'est un grand mal pour quelqu'un de malade [...].» Maunoir, Jean-Pierre, correspondance, ms., 27-Z-59, Musée d'histoire des sciences de Genève. - En revanche, sur ce même sujet, le poète Jean-Antoine Petit, dit Petit-Senn (1782-1870), écrit avec humour sous le titre «Quelques considérations sur la poussière», les lignes suivantes : «J'entends sans cesse autour de moi des gens mécontents de tout se plaindre amèrement de la poussière que font nos balayeurs en exerçant leur fonction dans le milieu du jour et dans les rues que l'on n'arrose pas. Je ne partage pas l'opinion des plaignants à cet égard ; j'aime la poussière [...]. » Journal de Genève, 28 juin 1827.

${ }^{21}$ Rapport des médecins «chargés par la Seigneurie d'examiner quelques sources d'eaux minérales trouvées à Collogny», daté du 15 août 1620 : «Nous soubs signés, Docteurs Médecins, Estans desmandés par No. et Honnoré Seigneur Monsieur Bitri, de la part de nos Magnifiques et très Honnorés Seigneurs, pour nous transporter à Cologni, aux fins de voir et examiner quelques sources d'eaux pour sçavoir si elles sont minérales et médicamenteuses, Certifions que le Lundi 14. Aoust nous avons esté tous ensemble audit Cologni, avoir odoré, veu la consistance et couleur comme aussi gousté de l'eau des sources qui nous ont esté montrées [...]. » Pièces des portefeuilles historiques No 2660, in Gautier, Léon, op. cit., p. 641.

${ }^{22}$ République et Canton de Genève. «Le Conseil d'Etat, vu l'art. 207 de l'ordonnance du Conseil fédéral du 26 mai 1936, sur les denrées alimentaires et les objets usuels, Arrête : Ch. I, Dispositions générales, Art. 1, Alinéa $1:$ Il est interdit de vendre des champignons n'appartenant pas aux espèces reconnues comestibles d'une manière certaine [...].» (Sic !). Réglement concernant la vente des champignons, du 17 janvier 1973.

${ }^{23}$ Maunoir, Jean-Pierre, correspondance, ms., 27-Z-59, Musée d'histoire des sciences de Genève.

${ }^{24}$ On sait comment l'annexion de Genève, décidée à Paris, fut imposée par la force armée, avec la complicité du résident de France Félix Desportes. Voici en quels termes le Direc- 
toire exécutif de la République française présentait cette annexion dans le «Traité de réunion de la République de Genève à la République Française », signé le 26 avril 1798 : "Article I, La République Française accepte le vœu des Citoyens de Genève pour leur réunion au Peuple Français. En conséquence, les Genevois, tant ceux qui habitent la ville et le territoire de Genève, que ceux qui sont en France et ailleurs, sont déclarés Français nés [...].»

${ }^{25}$ Département du Léman. Extrait des Registres de l'Administration municipale de la Commune de Genève, 23 frimaire, an sept, de la République française, une et indivisible.

\section{Summary}

After a short survey of the political, economical, and social situation of Geneva during the 16th century and of the situation of practical medicine during this period, the different stages of the genesis of the "Ordonnances sur la médecine" of 1569 are examined. The first ten articles of these ordinances, concerning the activities of physicians, are analysed in detail, while those concerning pharmacists and surgeons are left aside. The application of the ordinances and their revision in 1658 are commented upon. Eventually the evolution of the relations between physician, society, and state during the last centuries is shortly surveyed.

Dr. Roger Mayer

Archiviste de la Société Médicale de Genève

9, rue Michel-Chauvet

1208 Genève 